\title{
Open MRI validation of a hip model driven with subject-specific motion capture data in predicting anterior femoroacetabular clearance
}

\author{
Maryam Mohtajeb ${ }^{1,2}$, Jolanda Cibere ${ }^{3,4}$, Angelo Graffos ${ }^{5}$, Michelle Mony ${ }^{4}$, Honglin Zhang ${ }^{2}$, \\ Michael A. Hunt ${ }^{5,6}$ and David R. Wilson ${ }^{2,7^{*}}$ (1)
}

\begin{abstract}
Background: Cam and/or pincer morphologies (CPM) are potential precursors to hip osteoarthritis (OA) and important contributors to non-arthritic hip pain. However, only some CPM hips develop OA and/or pain, and it is not clear why. Anterior impingement between the femoral head/neck contour and acetabular rim during motion is a proposed pathomechanism. Understanding how activity and deformity combine to produce impingement may shed light on the causes of hip degeneration/pain. The objective of this study was to determine the accuracy of a subject-specific hip model driven by subject-specific motion data in predicting anterior impingement.

Methods: We recruited 22 participants with CPM (both with and without pain) and 11 controls. We collected subjectspecific 3D kinematics during squatting and sitting flexion, adduction, and internal rotation (FADIR) (an active and a passive maneuver, respectively, proposed to provoke impingement). We then developed 3D subject-specific hip models from supine 3T hip MRI scans that predicted the beta angle (a measure of anterior femoroacetabular clearance) for each frame of acquired kinematics. To assess the accuracy of these predictions, we measured the beta angle directly in the final position of squatting and sitting FADIR using open MRI scans. We selected the frame of motion data matching the static imaged posture using the least-squares error in hip angles. Model accuracy for each subject was calculated as the absolute error between the open MRI measure of beta and the model prediction of beta at the matched time frame. To make the final model accuracy independent of goodness of match between open MRI position and motion data, a threshold was set for least-squares error in hip angles, and only participants that were below this threshold were considered in the final model accuracy calculation, yielding results from 10 participants for squatting and 7 participants for sitting FADIR.
\end{abstract}

Results: For squatting and sitting FADIR, we found an accuracy of $1.1^{\circ}\left(0.8^{\circ}\right)$ and $1.3^{\circ}$ (mean (SD), and root mean squared error, respectively) and $0.5^{\circ}\left(0.3^{\circ}\right)$ and $0.6^{\circ}$, respectively.

Conclusion: This subject-specific hip model predicts anterior femoroacetabular clearance with an accuracy of about $1^{\circ}$, making it useful to predict anterior impingement during activities measured with motion analysis.

Keywords: Biomechanics, Hip, Femoroacetabular impingement, Mechanics, Model, Motion analysis

*Correspondence: dawilson@interchange.ubc.ca

${ }^{7}$ Department of Orthopaedics, University of British Columbia, 7/F, 2635

Laurel Street, Vancouver, BC V5Z1M9, Canada

Full list of author information is available at the end of the article

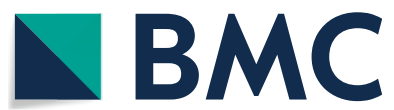

(c) The Author(s) 2021. Open Access This article is licensed under a Creative Commons Attribution 4.0 International License, which permits use, sharing, adaptation, distribution and reproduction in any medium or format, as long as you give appropriate credit to the original author(s) and the source, provide a link to the Creative Commons licence, and indicate if changes were made. The images or other third party material in this article are included in the article's Creative Commons licence, unless indicated otherwise in a credit line to the material. If material is not included in the article's Creative Commons licence and your intended use is not permitted by statutory regulation or exceeds the permitted use, you will need to obtain permission directly from the copyright holder. To view a copy of this licence, visit http://creativecommons.org/licenses/by/4.0/. The Creative Commons Public Domain Dedication waiver (http://creativeco mmons.org/publicdomain/zero/1.0/) applies to the data made available in this article, unless otherwise stated in a credit line to the data. 


\section{Introduction}

Cam and pincer morphologies (CPM), which describe, respectively, an aspherical femoral head/head-neck junction and acetabular over-coverage, are potential precursors to hip osteoarthritis [1-8] and important contributors to non-arthritic hip pain [9-12]. However, only a subset of hips with CPM develops symptoms, which are primarily position-related pain in the hip and/or groin [13]. It is not clear which factors besides CPM are associated with symptoms and/or osteoarthritis. Impingement between the femoral head-neck junction and acetabular rim in extreme hip rotations is a proposed pathomechanism in femoroacetabular impingement (FAI) [1]. Impingement is sometimes assessed intraoperatively, but identifying impinging regions, especially arthroscopically, is technically challenging, and findings can be different from actual impingement occurring during in-vivo hip articulation in daily activities [14].

Biomechanical studies have examined hip rotational kinematics and kinetics during level walking [15-17] and other functional activities [18-21] in participants with CPM. A systematic review and meta-analysis [22] of these studies concluded that CPM hips with symptoms have lower peak hip extension angle, peak hip internal rotation angle, and external rotation hip torque during walking compared to control hips. For other activities, the review concluded that CPM hips with symptoms squatted to a lower depth compared to control hips with no differences in peak hip angles in all three planes at maximum squat depth. Evidence regarding the pelvic range of motion and other studied tasks like stair ascent was insufficient for any conclusion. It is not clear whether these observed alterations in hip kinematics and kinetics are directly caused by impingement or whether they are related to other mechanisms because direct measurement of femoroacetabular relationships cannot be made using these methods.

Impingement has been studied using finite element modeling of the hip to calculate mechanical stresses within the joint. Maximum shear stresses (MSS) in the acetabular cartilage and underlying bone in standing and maximum squat positions were calculated in a finite element study using hip models from two participants with severe cam deformity and two matched controls [23]. Inputs for each finite element model, including hip angles and net hip joint forces, were acquired from each participant's motion capture data during a squat test. While similar cartilage MSS peaks were found in cam hips for standing (3.9 MPa) and squatting (3.6 MPa), MSS peaks of the underlying bone in cam hips were up to 4.7 times higher for squatting compared to standing. Further work is required to develop a robust finite element model of the hip that can predict impingement [24]. While the advantage of finite element studies is that they predict mechanical stresses, these models require many inputs (e.g., material properties, geometry, movement) that make validation and studies of large numbers of participants difficult.

Other studies have combined imaging and joint movement data to predict joint angles where contact is reached and/or contact locations on the femur and acetabulum [25-28]. One study assessed bony impingement points for simulated movements applied to 3D hip models constructed from $\mathrm{CT}$ data from 10 symptomatic cam hips, 10 asymptomatic cam hips, and 10 control hips [25]. In symptomatic cam hips, bony impingement occurred at significantly reduced internal rotation compared to asymptomatic cam hips and control hips. Unexpectedly, a significant number of identified impingement points occurred at the anteromedial part of the femoral neck rather than at the cam lesion. Impingement at the anterior part of the femoral neck was also observed for healthy control hips [25]. Another study that assessed bony impingement for applied simulated movements in models of 8 symptomatic CPM hips found that impingement regions could not be predicted using measurements of hip bony architecture: zones of initial contact did not correspond to the regions with minimum femoral headneck offset in every case [27]. One limitation of this modeling approach is that it didn't consider subject-specific kinematics data required to describe the subject-specific differences due to the unique neuromuscular, capsuloligamentous, and bony contributions to the motion of each patient. This limitation was addressed in a study using subject-specific motion data collected using a magneticbased kinematics system and applied to 3D models of 13 cam hips [28], which found abutment of different regions of cam morphology against the anterosuperior quadrant of the acetabulum during various maneuvers. A limitation of these modeling studies is that, because few direct measurements of three-dimensional hip anatomy have been made at high hip rotation angles, these models have not been validated fully. None of these studies used subject-specific kinematics data collected using motion capture systems that are the current standard (state of the art) for studying human kinematics.

Our understanding of the role of activity in hip impingement might be improved by using subject-specific motion data to drive models with subject-specific hip anatomy from imaging. The motion data could include a large range of dynamic daily activities. Validating such models require three-dimensional visualization of the hip in a range of postures.

Research Question: What is the accuracy of a subjectspecific hip model driven by subject-specific motion data in predicting anterior femoroacetabular clearance 
(impingement) during squatting and sitting flexion, adduction, and internal rotation (FADIR) maneuvers?

\section{Method}

To assess the accuracy of subject-specific hip model predictions of anterior femoroacetabular clearance for squatting and sitting FADIR, we compared model predictions to direct measurements of anterior femoroacetabular clearance made using open MRI scans of the hips in the same postures. These two maneuvers require extreme hip angles, which are proposed to provoke impingement. Squatting represents an active impingement provoking maneuver while sitting FADIR represents a passive impingement provoking posture.

\section{Participants}

We recruited 33 participants aged 28-56years old, including 9 with cam and/or pincer morphology and with pain $(\mathrm{CPM}+), 13$ with cam and/or pincer morphology and without pain (CPM-), and 11 controls. All participants were recruited from the original Investigation of Mobility, Physical Activity, and Knowledge Translation in Hip Pain (IMPAKT-HIP) cohort.

The IMPAKT-HIP cohort is a population-based sample of 500 Caucasian people recruited through random-digit dialing of households in greater Vancouver [29, 30]. In all participants, morphology of the femoral head-neck contour and acetabular coverage were assessed using supine Dunn view radiographs of the hip and standardized weight-bearing anteroposterior radiographs of the pelvis [31]. Hips were identified as having cam morphology if the alpha angle [32] was greater than $55^{\circ}$ on the Dunn view radiograph [33]. Hips were identified as having pincer morphology if they had a lateral center edge (LCE) angle $[34,35]$ greater than $40^{\circ}$ and/or a positive crossover sign on the anteroposterior radiograph [31]. Hips identified as having both cam and pincer morphologies were classified as mixed. The presence of hip pain was defined as participant-reported pain in the groin and/or upper thigh lasting for 6 weeks or more and/or for 3 or more episodes during the past 12 months. This definition was designed to exclude pain due to soft tissue injuries/ deficiencies and identify pain originating only from the hip. The study hip was defined as the hip with radiographic CPM. If CPM was present in both hips, then the hip with more severe pain was defined as the study hip. If equal or no hip pain was reported, the study hip was randomly selected. Groups were defined as follows: CPM+ hips were positive for the presence of pain and had at least one radiographic CPM; CPM- hips were negative for the presence of pain and positive for at least one radiographic CPM; Controls were negative for the presence of pain and had no radiographic CPM. Recruitment and screening for the original IMPAKT-HIP study spanned 1.5 years.

For our current study, which occurred a mean (SD) of 5.7(0.5) years after the original IMPAKT contact, phone screening was completed to recruit participants. The same definitions as the original IMPAKT study were used for CPM, hip pain, and the study hip. New exclusion criteria were considered for the current study, including previous lower limb surgeries, injuries, or any neurological conditions that affected everyday recreational or sporting activities over the past 12 months, a history of any inflammatory or autoimmune diseases, avascular necrosis of the hip, planned or previous lower limb joint replacement, or physician-diagnosed lower limb joint osteoarthritis. The Clinical Research Ethics Board of the University of British Columbia approved the study, written informed consent was obtained from all participants, and the study was conducted in accordance with the Declaration of Helsinki.

\section{Motion analysis}

We collected 3D kinematics data of each participant during squatting and sitting FADIR maneuvers at $120 \mathrm{~Hz}$ using a fourteen-camera motion capture system (Motion Analysis Corporation, Santa Rosa, CA). Fifty passive retroreflective markers applied to various bony landmarks were used for motion tracking. Bilateral bony landmarks included the acromioclavicular joint, posterior superior iliac spine (PSIS), iliac crest, anterior superior iliac spine (ASIS), greater trochanter, anterior thigh, lateral and medial femoral epicondyle, anterior shank, lateral and medial malleoli, posterior calcaneus, medial aspect of the head of the 1st metatarsal bone, dorsal aspect of the head of the 2nd metatarsal bone, and the lateral aspect of the head of the 5th metatarsal bone. Other bony landmarks included vertebra $\mathrm{C}_{7}$, vertebra $\mathrm{T}_{10}$, right scapula, and the sternal notch. Finally, cluster plates of 4 markers were applied bilaterally on the shanks and thighs to track the movements of their respective segments during the squatting and sitting FADIR maneuvers. A static standing trial with the same foot distance and orientation as the open MRI standing posture was then conducted. After the static standing trial was completed, the medial epicondyle and malleoli markers were removed for the squatting and sitting FADIR trials.

\section{Squatting}

Participants were positioned with their feet oriented anteriorly and with the most lateral aspects of the toes $22 \mathrm{~cm}$ apart and their arms crossed across their chest. They squatted as deeply as they could without rotating or lifting any part of their feet (Fig. 1 (a)). Maximum squat depth was measured for each participant from the 

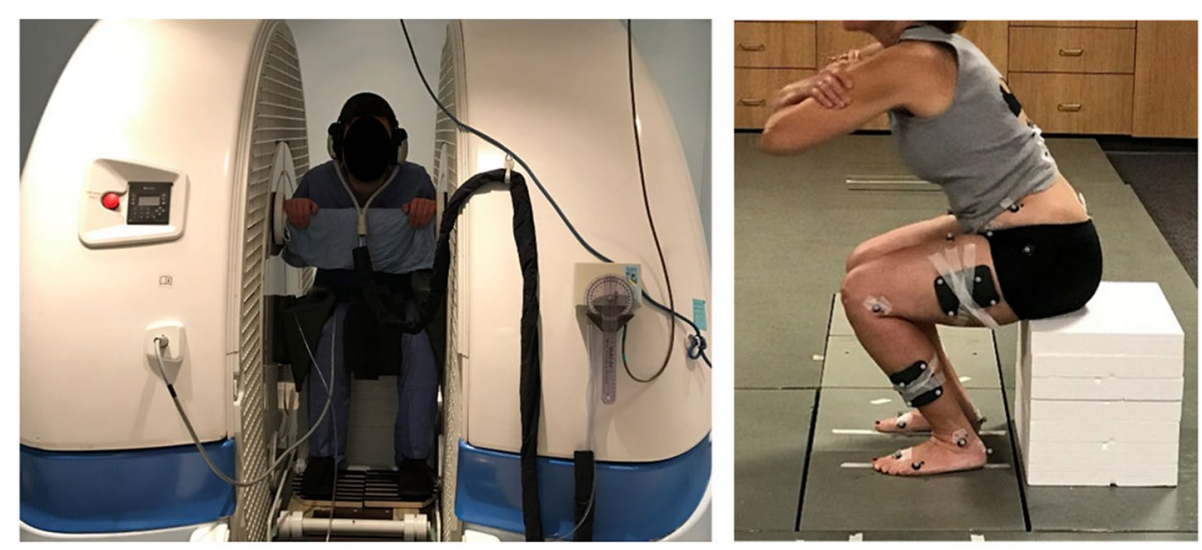

(a) Squatting
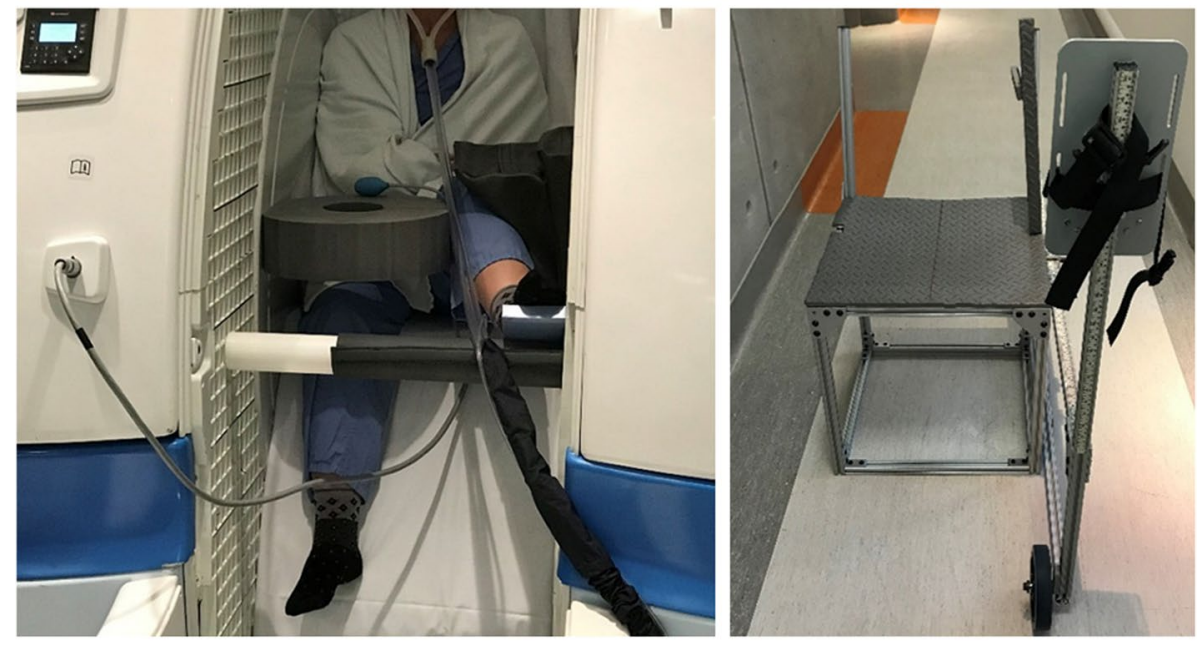

(b) Sitting FADIR

Fig. 1 Positioning at the MROpen scanner and motion capture lab for (a) squatting and (b) sitting FADIR

ground to the lowest part of the buttocks. Motion data for 5 trials were recorded for each participant. The toe distance restriction was applied because the final position of the squatting maneuver performed in the motion analysis lab needed to be replicated in the open MRI scanner during the scanning and was dictated by the open MRI scanner bore dimensions and the necessity of positioning the study hip at the scanner isocenter.

\section{Sitting FADIR}

For sitting FADIR, the participant's study side hip was moved into the FADIR pose using a chair designed to match the bore and chair dimensions of our open MRI scanner (Fig. 1 (b)). The horizontal distance between the mid-point of the chair seat and the most lateral margin of the chair foot holder is equal to the horizontal distance between the mid-point of the open MRI chair and open MRI wall. Participants were positioned with their study hip in the middle of the chair while their foot was secured in the chair foot holder.

The chair design allowed each participant's hip and foot to be positioned and constrained as it was within the open MRI scanner (Fig. 1 (b)) for the same posture. The study hip was flexed and then adducted and internally rotated to the maximum limit the participant could tolerate for the duration of scanning (about $30 \mathrm{~min}$ ) using foot displacement in the horizontal and vertical direction and then moving the knee toward the body mid-line. Motion data for 5 trials were recorded for each participant.

\section{D hip models}

To develop 3D subject-specific hip models, we scanned each participant's study hip in supine using a sequence optimized for hip cortical bone visualization in a 3T MRI scanner (Achieva, Philips, Eindhoven, Netherlands) (Table 1). 3T scanning of the hips was performed a mean (SD) of $4.2(0.54)$ years prior to the current study and 
Table 1 Sequence details of 3T MRI hip scans used for developing 3D hip models and MROpen hip/pelvis/knee scans used for defining a hip joint coordinate system in supine/standing and calculating hip angles in squatting/sitting FADIR in the MROpen

\begin{tabular}{|c|c|c|c|c|}
\hline & Sequence & Matrix & $\begin{array}{l}\text { FOV } \\
\text { (field of view) }\end{array}$ & Slice Thickness \\
\hline 3T MRI Hip scans & $\begin{array}{l}\mathrm{TSE}^{1} \\
\mathrm{TE}^{2} / \mathrm{TR}^{3}=10 \mathrm{~ms} / 776.7 \mathrm{~ms}\end{array}$ & $512 \times 512$ & $20 \mathrm{~cm} \times 20 \mathrm{~cm}$ & $2 \mathrm{~mm}$ \\
\hline MROpen Hip Alpha Plane scans & $\begin{array}{l}\mathrm{GFE}^{4} \text { short TE, } \\
\mathrm{TE} / \mathrm{TR}=8 \mathrm{~ms} / 443 \mathrm{~ms}\end{array}$ & $256 \times 256$ & $25 \mathrm{~cm} \times 25 \mathrm{~cm}$ & $2.5 \mathrm{~mm}$ (0.5 mm gap) \\
\hline MROpen Hip Sagittal Scans & $\begin{array}{l}\text { GFE, short TE sequence, } \\
T E / T R=8 \mathrm{~ms} / 627 \mathrm{~ms}\end{array}$ & $256 \times 256$ & $25 \mathrm{~cm} \times 25 \mathrm{~cm}$ & $2.5 \mathrm{~mm}$ (0.5 mm gap) \\
\hline MROpen Pelvis Axial Scans & $\mathrm{GFE}, \mathrm{TE} / \mathrm{TR}=12 \mathrm{~ms} / 370 \mathrm{~ms}$ & $256 \times 256$ & $30 \mathrm{~cm} \times 30 \mathrm{~cm}$ & $2.5 \mathrm{~mm}$ (0.5 mm gap) \\
\hline MROpen Knee Axial Scans & $\mathrm{GFE}, \mathrm{TE} / \mathrm{TR}=8 \mathrm{~ms} / 650 \mathrm{~ms}$ & $256 \times 256$ & $20 \mathrm{~cm} \times 20 \mathrm{~cm}$ & $2.5 \mathrm{~mm}$ (0.5 mm gap) \\
\hline
\end{tabular}

${ }^{1}$ Turbo spin echo, ${ }^{2}$. Echo Time, ${ }^{3}$. Repetition Time, ${ }^{4}$. Gradient Field Echo

spanned 1.08 years. Subject-specific 3D models (point clouds) of the femur and acetabulum were developed by segmenting these bones manually from 3T MRI scans using Analyze software (AnalyzeDirect, Inc., Overland Park, KS, US).

\section{Open MR imaging}

We scanned each participant's study hip in supine, standing, squatting, and sitting FADIR poses with an upright open MRI scanner (MROpen, Paramed, Genoa, Italy) to a) measure impingement directly and b) measure hip angles. To measure impingement, we acquired scans in planes parallel to the femoral neck and perpendicular to the femoral neck and femoral shaft axes (alpha plane) (Table 1) in squatting and sitting FADIR. For hip angles, we acquired images in the sagittal plane (Table 1) for supine, squatting, and sitting FADIR. We also scanned each participant's pelvis and study side knee in supine to define a hip joint coordinate system.

We applied the following protocols for the squatting and sitting FADIR postures in the MROpen.

\section{Squatting}

The MROpen bed was adjusted, and several foam pads were placed on the bed to replicate the squat depth measured in the motion analysis lab for each participant. Participants were positioned so that the top foam was touching the buttocks to prevent participant motion and associated movement artifact. Foot orientation and position from the motion analysis lab were replicated in the scanner using a pair of sandals attached to a wooden plate. Several foam pads were put around the participants' knees to minimize movement artifact (Fig. 1 (a)).

\section{Sitting FADIR}

Participants were positioned in the scanner chair with the study hip at the center of the scanner. The hip was flexed, adducted, and internally rotated to the same posture used in the motion analysis study. The foot was secured against the MROpen scanner wall using a support bar. The knee was supported with several foam pads to minimize motion artifact (Fig. 1 (b)).

\section{Hip angle measurement from MROpen}

To calculate hip joint angles from the MROpen images, we first defined hip joint coordinate systems according to the International Society of Biomechanics (ISB) recommendations [36] using the supine scans. To define the hip joint coordinate system, a Cartesian coordinate system was first defined for both the femur and pelvis. The pelvis coordinate system was defined using the right and left anterior superior iliac spines (ASIS) and right and left posterior superior iliac spines (PSIS). Orientations of pelvis coordinate system unit vectors were as follows: Z-axis: parallel to the line connecting the right and left ASIS pointing laterally; X-axis: parallel to a line lying in the plane defined by right and left ASIS and the midpoint of the right and left PSIS, perpendicular to the Z-axis, pointing anteriorly; Y-axis: cross product of $\mathrm{X}$ and $\mathrm{Z}$ pointing superiorly. The origin of the pelvis coordinate system was defined as the center of the best fit sphere to the acetabulum lunate surface. The femur coordinate system was defined using medial and lateral femoral epicondyles and the center of the best fit sphere to the femoral head surface. Orientations of the femur coordinate system unit vectors were as follows: y-axis: the line joining the midpoint between the medial and lateral femoral epicondyles and the center of the best fit sphere to the femoral head pointing superiorly; z-axis: the line lying in the plane defined by the center of the best fit sphere to the femoral head and the medial and lateral femoral epicondyles perpendicular to $y$, pointing laterally; $x$-axis: cross product of $y$ and $\mathrm{z}$ pointing anteriorly. The orientations of the hip joint coordinate system unit vectors are as follows: $\mathbf{e}_{\mathbf{1}}$ : Z-axis 


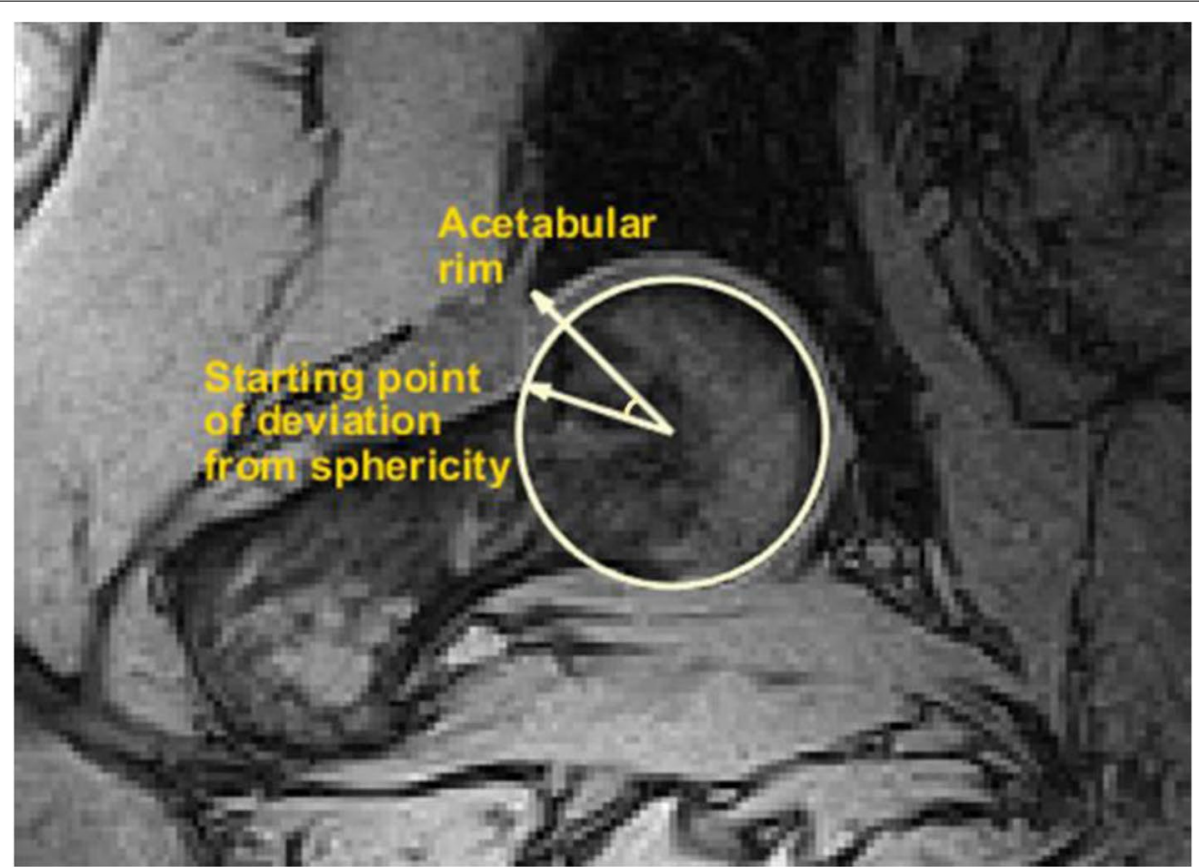

Fig. 2 Illustration of beta angle in a control hip at the squatting posture

of the pelvis coordinate system fixed to the pelvis (flexion/extension); $\mathbf{e}_{2}: y$-axis of the femur coordinate system fixed to the femur (internal/external rotation); $\mathbf{e}_{3}$ : cross product of $\mathbf{e}_{1}$ and $\mathbf{e}_{2}$, which is the floating axis (abduction/adduction). The origin of the hip coordinate system was considered the same as the femur coordinate system origin. Bony landmarks, including right and left ASIS/ right and left PSIS, and medial/lateral femoral epicondyles were identified from the supine scans of the pelvis and knee, respectively. The femoral head surface and acetabular lunate surfaces were segmented from the supine sagittal scans of the hip.

To calculate hip joint angles from the MROpen images of the squatting and sitting FADIR poses, 3D models of the femur and acetabulum in the supine, squatting, and sitting FADIR postures were created by segmenting sagittal scans of the hip for these postures. 3D models in supine were registered to 3D models for the squatting and sitting FADIR postures using the finite iterative closest point (ICP) algorithm [37]. Hip joint angles in each posture were calculated using the Grood and Suntay convention [38] applied to the hip joint coordinate system.

\section{Model validation}

The primary outcome variable was the beta angle, which defines clearance between the femoral head/neck junction and the acetabular rim [39]. The beta angle is measured on the same imaging plane as the alpha angle and is defined as the angle between a line drawn from the femoral head center to the most lateral bony margin of the acetabular rim and a second line drawn from the center of the femoral head to the starting point of deviation from sphericity in the femoral head-neck contour (Fig. 2). There is a significant association between negative beta angle and elevated acetabular rim contact pressures [40].

We calculated hip joint angles from the motion analysis data using the same joint coordinate system. The position of the joint coordinate system (found in supine) in the standing posture was determined by using transformation matrices found by registering 3D hip models in supine to 3D hip models in standing created from sagittal hip scans in supine and standing, respectively. The locations of the motion analysis markers in the identical (standing) posture used in the MROpen scanner were determined. Given the location of the joint coordinate system and the motion analysis markers in this reference standing posture, the location of the joint coordinate system for any subsequent frame of motion analysis data could be calculated, and joint angles could be determined using the Grood and Suntay convention [38].

Hip position for the squatting and sitting FADIR postures imaged in the MROpen scanner was matched to the motion analysis data by choosing the frame of motion analysis data that yielded the minimum least-squares error between hip joint angles in the MROpen and motion analysis data. 
For the squatting and sitting FADIR postures, we compared the model prediction of the beta angle to the direct measurement of the beta angle on the MROpen scan. We determined the hip joint angles for the squatting posture in the MROpen scanner and then identified the frame of squatting from the motion analysis data that best matched these joint angles (i.e., with least-squares error in hip joint angles). Femur and acetabulum 3D models segmented from the 3T scans were positioned to this identified time frame of motion sequence using calculated transformation matrices from the supine posture (corresponding to the 3T image acquisition) to the relevant frame of motion analysis data. The femur's position was adjusted to match the femur and acetabulum centerto-center distance calculated from the 3T MRI scans. The orientation of the alpha plane was determined, and a series of planes parallel to the alpha plane and spaced $2.5 \mathrm{~mm}$ apart were passed through the positioned 3D model of the hip to replicate MROpen slices. The intersection of these planes with the hip 3D models was found as the points within $0.5 \mathrm{~mm}$ of the defined planes. The beta angle was calculated for each plane. The minimum beta angle (for all planes) was found and compared to the minimum beta angle measured from the MROpen scans.

\section{Statistical analysis}

We calculated each subject-specific model accuracy as the absolute difference between the beta angle calculated from the hip model and the beta angle measured directly from the MROpen scans. Final model accuracy was described with the mean (SD) (and root mean squared) of subject-specific model accuracies.

We assessed the relationship between the least-squares error in hip angles and subject-specific model accuracy using the Pearson correlation coefficient (and intraclass correlation coefficient (ICC)) to better understand how model accuracy is related to how well the positions from the motion analysis data and the MROpen data are aligned.

To exclude data points where the accuracy would be affected by the difference in hip position between the MROpen posture and the motion analysis posture, we set a threshold for the least-squares error of hip angles, and only considered participants that were below this threshold in the final model accuracy calculation. This threshold was defined by including participants with the lowest least-squares error in hip angles until there was no statistically significant relationship between the least-squares error in hip angles and subject-specific model accuracy. For squatting, we had to include participants with less than $10 \%$ mean least-squares error in hip angles (10 participants) to reach no significant correlation $(r=0.47$, $p=0.17$ ) between the least-squares error of hip angles and subject-specific model accuracy $(\mathrm{ICC}=0.041)$. For sitting FADIR, we had to include participants with less than 5\% mean least-squares error in hip angles (7 participants) to reach no significant correlation $(r=0.63$, $p=0.12$ ) between the least-squares error of hip angles and subject-specific model accuracy $(\mathrm{ICC}=0.0071) \quad(3$ participants who met the threshold criteria for the sitting FADIR were excluded from the final accuracy calculation because either they hadn't completed the 3T MRI scanning or had very low-quality scans).

We assessed the relationship between model accuracy and participant body mass index (BMI) using the Pearson correlation (and ICC) to investigate how BMI affects the amount of skin-mounted marker movement relative to the hip bones and model accuracy.

\section{Results}

For squatting, the mean absolute error (SD) and root mean squared error (RMSE) between the model prediction of beta angle and the direct measure of beta angle from the MROpen were $1.1^{\circ}\left(0.8^{\circ}\right)$ and $1.3^{\circ}$, respectively (Table 2) (Fig. 3 (a)). We found no statistically significant correlation $(\mathrm{r}=-0.25, p=0.48)$ between the model accuracy and participant $\mathrm{BMI}$ for the squat $(\mathrm{ICC}=0.047)$.

For sitting FADIR, the mean absolute error (SD) and root mean squared error (RMSE) between the model prediction of beta angle and the direct measure of beta angle from the MROpen was $0.5^{\circ}\left(0.3^{\circ}\right)$ and $0.6^{\circ}$, respectively (Table 3) (Fig. 3 (b)). We found no statistically significant correlation $(\mathrm{r}=0.51, p=0.24)$ between the model accuracy and participant BMI for sitting FADIR $(\mathrm{ICC}=0.004)$.

\section{Discussion}

In this study, we assessed the accuracy of a subject-specific hip model combined with motion analysis data in predicting the beta angle, a measure of hip clearance, by comparing model predictions to direct measurements made using open MRI. We found an accuracy of $1.1^{\circ}$ for squatting and an accuracy of $0.5^{\circ}$ for sitting FADIR.

This simple model allows subject-specific assessment of hip clearance using motion data combined with a supine and standing MRI scan of the hip and a supine scan of the pelvis and knee to identify required bony landmarks for defining the hip joint coordinate system. Our accuracy figures for beta angle prediction are useful for planning and interpreting studies using this model. Our correlation results for BMI show that his model is not affected by participant BMI within the range of BMIs that we tested. The model in this study can predict impingement without requiring assumptions such as simplified anatomy or averaged material properties for the bone or soft tissues. 


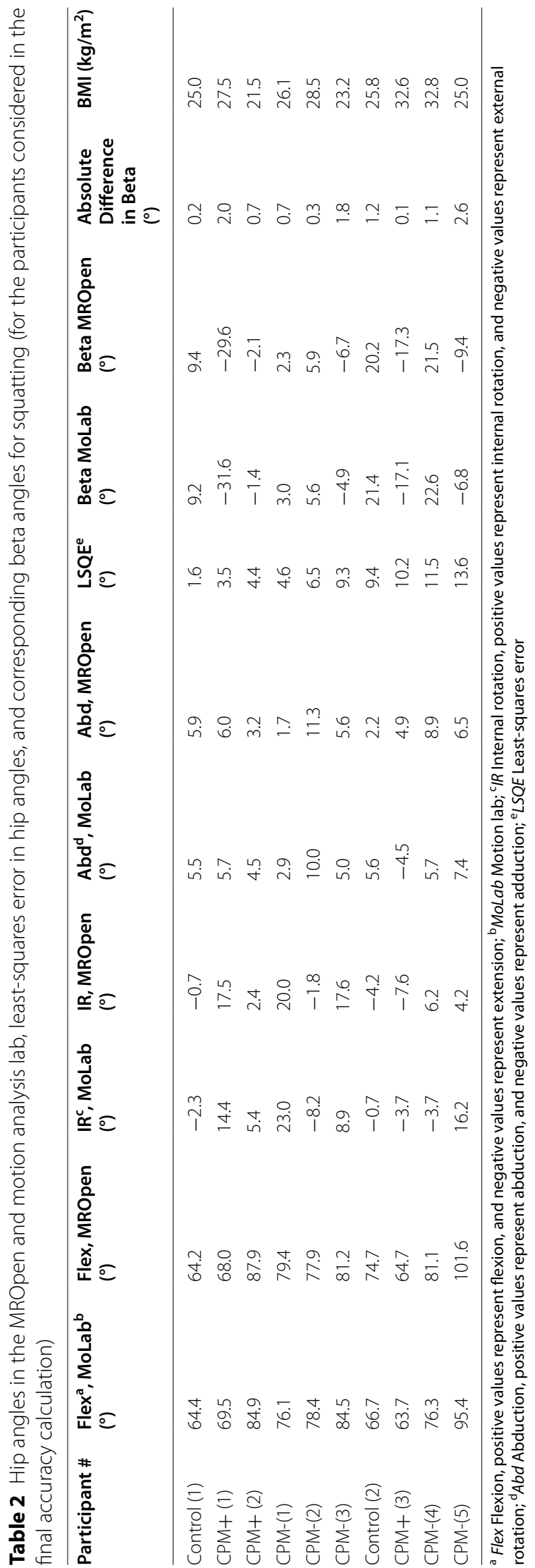




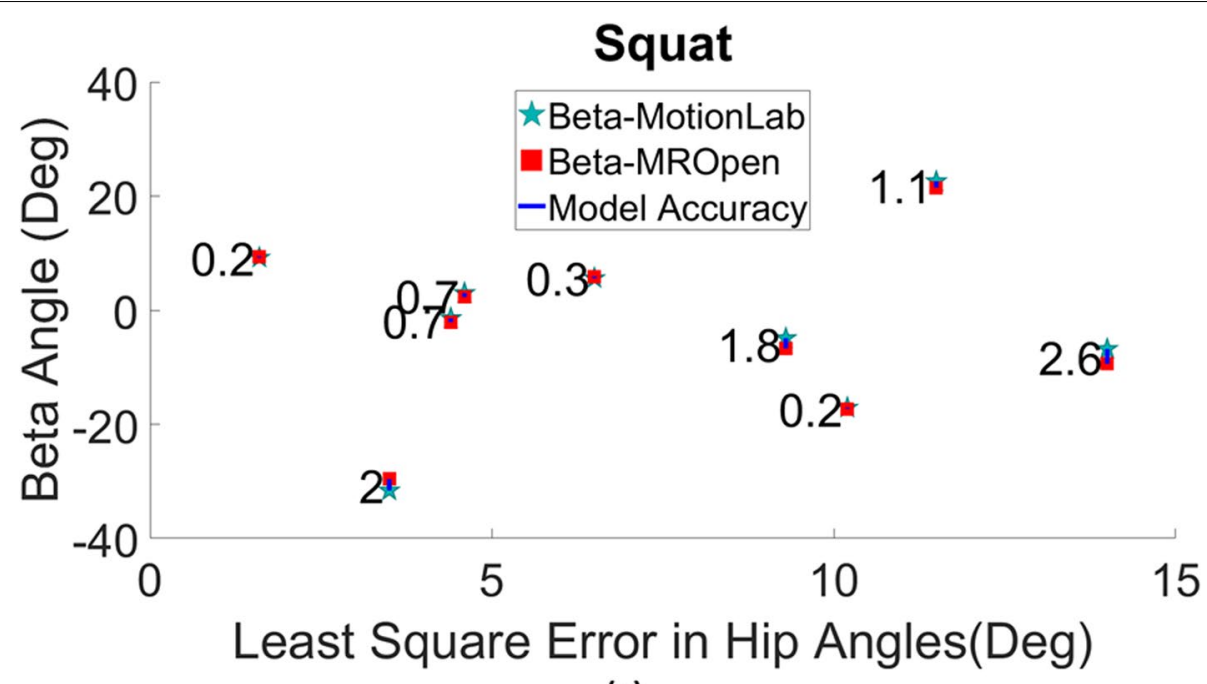

(a)

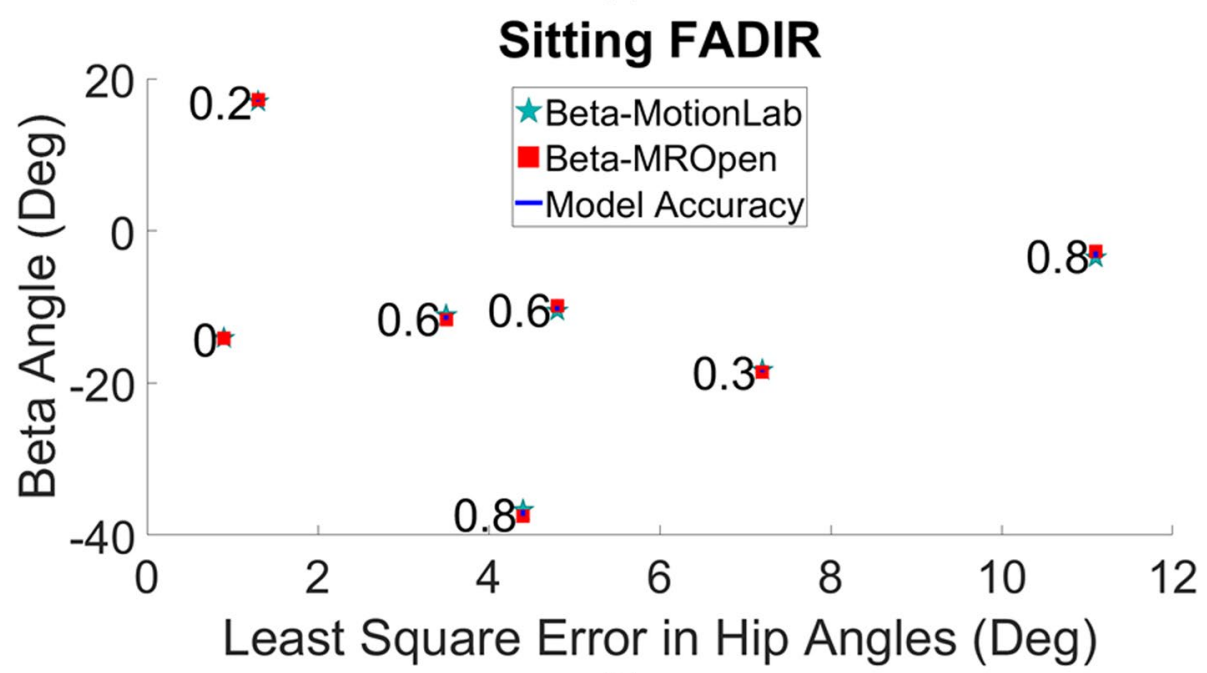

(b)

Fig. 3 Beta measured from MROpen scans and beta calculated from subject-specific hip models (motion lab) and their absolute differences (label numbers) as the subject-specific model accuracy in predicting beta angle for (a) squatting and (b) sitting FADIR; in terms of the least-squares error in hip angles (for participants considered in the final accuracy calculation)

The accuracy of our model is comparable to the accuracy of a combined dual fluoroscopy and model-based tracking approach used for studying kinematics of $3 \mathrm{CPM}$ hips with symptoms and 6 control hips [41, 42]. This study reported a bias and precision of less than $1^{\circ}$ using an ex-vivo cadaver study. Although highly accurate, the need for ionizing radiation and the somewhat limited imaging region associated with dual fluoroscopy make it impractical for assessing a large range of activities.

This model has the potential to provide valuable data that support and enhance what has been learned from finite element models of the hip [23, 43-45]. In these models, the geometry of hip structures, including the femur, acetabulum, cartilage, and labrum (if considered), were modeled using either subject-specific imaging data or more simplified geometry. Most of these studies assumed isotropic, linear elastic behavior for the cartilage and labrum; however, one study modeled the biphasic behavior of cartilage. Subject-specific kinematics and kinetics data were assumed in some but not all of these studies. Regardless of the simplifications and sophistication of the modeling approaches, all these studies showed that intrusion of the non-spherical femoral head-neck contour into the acetabulum (impingement) corresponds to elevated joint stresses. The increased stresses were larger at higher hip angles (i.e., reaching the terminal 


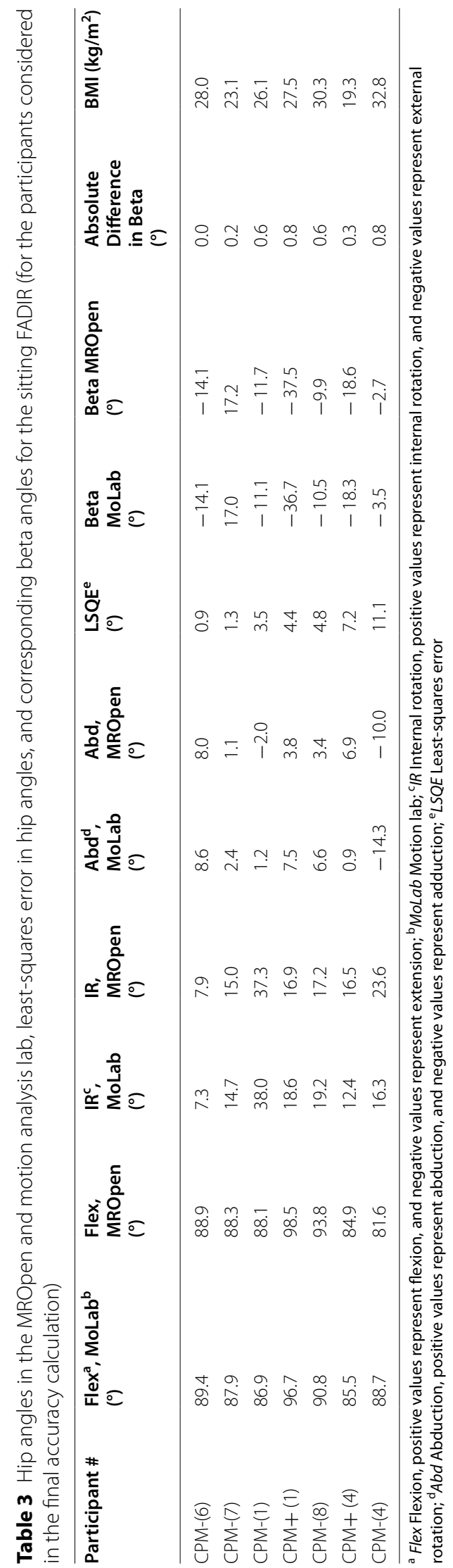


position of the studied maneuvers). The model from the current study can be used to predict when and to what extent this intrusion is happening during dynamic activities.

An advantage of our model over the dynamic CT models that are currently used clinically to predict impingement is that our model uses subject-specific kinematics data. Many dynamic CT models [25-27] rotate the femur relative to the acetabulum using generalized hip motions until femoroacetabular collision is detected, which is defined as the end of the hip range of motion, and the points of contact are considered as impingement regions. Biomechanical studies have shown that there are differences in hip and pelvic kinematics between hips with FAI syndrome and pain-free control hips, which demonstrates the importance of using subject-specific hip kinematics to predict impingement. One dynamic CT model [28] used subject-specific kinematics, but only from passive maneuvers of the hip. Further, we are not aware of direct validation of impingement predicted by these CT simulations against actual measures of impingement in-vivo.

This model could be implemented in clinical practice, but two essential steps would likely be required to make the procedure less time-consuming. First, we would modify the procedure to estimate the hip coordinate system from the external motion markers, which would enable us to run the model without the standing scan of the hip. This would require quantifying the effect of defining the hip coordinate system using the external motion markers rather than actual anatomical landmarks on the model accuracy. Second, we would automate the segmentation of hip bones from the MRI scans, which would be much faster than the manual segmentation used in this study. This would require quantifying the effect of automatic segmentation on the accuracy of the model.

One strength of the study is that we assessed model accuracy in-vivo using the population that this model was developed to study rather than in cadavers or control hips. Hip kinematics are influenced by hip morphology and pain status, and our study included CPM+, CPM-, and normal hips. Unfortunately, we did not have sufficient numbers of participants in each group to compare our accuracy findings between these groups. A second strength is that we assessed model accuracy during both passive (sitting FADIR) and active (squatting) maneuvers. Soft tissue behavior might be different during an active maneuver than a passive maneuver, which might affect hip kinematics and, therefore, model predictions. A final strength is that hip angles during the motion analysis maneuvers were calculated using the hip joint coordinate system built through the actual bony landmarks identified from the MROpen scans. This method is in contrast with the more widely used approach of considering retroreflective motion capture markers as actual bony landmarks and estimating the femoral head center using these external markers, which can be inaccurate because of the soft tissue layer between the external retroreflective markers and actual bony landmarks as well as errors in locating the bony landmarks.

One limitation of our study is that we validated this model for only two postures. However, these postures were chosen because they are likely to place the hip in an impinging position and therefore represent important positions for a model that assesses impingement. A second limitation is that the $3 \mathrm{~T}$ scans of the hips were acquired a mean (SD) of $4.2(0.54)$ years prior to our current study. This limitation is unlikely to affect our findings because the $3 \mathrm{~T}$ scans were only used to describe the bony anatomy, and it has been shown that femoral head-neck anatomy in cam hips and acetabular coverage in dysplastic hips does not change over 5 years [46] and 20 years [47]. Another limitation of our study is that the 3T scans had a relatively large slice thickness $(2 \mathrm{~mm})$. An isotropic high-resolution scan of the hip could make the 3D representation of the hip joint closer to the actual morphology. A further limitation is that we did not assess the accuracy of this model for the more widely used case where the hip joint coordinate system is defined using external retroreflective motion capture markers instead of bony landmarks acquired from MRI. Finally, we assessed accuracy in only 10 participants for the squat and 7 participants for the sitting FADIR due to the challenges of closely matching MROpen to motion analysis postures, which, though somewhat limited, is higher than many other validation studies.

\section{Conclusion}

We conclude that this subject-specific hip model driven by subject-specific motion data predicts beta angle (anterior femoroacetabular clearance) with an accuracy of about $1^{\circ}$, which makes it useful for predicting anterior impingement during activities measured with motion analysis.

\footnotetext{
Abbreviations

OA: Osteoarthritis; CPM: Cam and/or pincer morphologies; FADIR: Flexion, adduction, and internal rotation; RMSE: Root mean squared error; FAI: Femoroacetabular impingement; MSS: Maximum shear stresses; CPM+ : Cam and/or pincer morphology with pain; CPM-: Cam and/or pincer morphology without pain; IMPAKT-HIP: Investigation of Mobility, Physical Activity, and Knowledge Translation in Hip Pain; MROpen: Open MRI scanner; PSIS: Posterior superior iliac spine; ASIS: Anterior superior iliac spine; ISB: International Society of Biomechanics; ICC: Intra-class correlation coefficient.
} 


\section{Acknowledgements}

We acknowledge the contribution of the entire IMPAKT-HIP research team to recruiting the participants in this study.

\section{Authors' contributions}

MMoh was the lead investigator and responsible for the study concept and design, data collection, data analysis, statistical analysis, presenting the data, interpreting the results, and manuscript writing. JC supervised the recruitment part of the study and recruited the IMPAKT-HIP cohort originally. AG helped with motion data collection and post-processed the data. MMon re-recruited participants from the IMPAKT-HIP cohort through phone screening. HZ contributed to the open MRI sequence development. MH was a co-investigator on the original grant used for conducting this study and contributed to the study concept and design. DW contributed to the study concept and design, reviewed the manuscript and provided supervision. DW was the primary investigator on the original grant used for conducting this research. All authors have read and approved the final submitted manuscript and have agreed both to be personally accountable for the author's own contributions and to ensure that questions related to the accuracy or integrity of any part of the work, even ones in which the author was not personally involved, are appropriately investigated, resolved, and the resolution documented in the literature.

\section{Funding}

This study was supported by an Arthritis Society of Canada Strategic Operating Grant (SOG-15-297), a Canadian Institutes of Health Research Project Grant (\#148828), a Natural Sciences and Engineering Research Council of Canada Discovery Grant (\#05246), and a Canadian Institutes of Health Research Team Grant (PAF-107513). The funding bodies played no role in the design of the study and collection, analysis, and interpretation of data and in writing the manuscript. The authors have no professional or financial affiliations that may be perceived to have biased the presentation to disclose.

\section{Availability of data and materials}

The datasets used and/or analysed during the current study are available from the corresponding author on reasonable request.

\section{Declarations}

\section{Ethics approval and consent to participate}

The Clinical Research Ethics Board of the University of British Columbia approved the study, written informed consent was obtained from all participants, and the study was conducted in accordance with the Declaration of Helsinki. The Clinical Research Ethics Board certificate number is H12-01971-A028.

\section{Consent for publication}

Not applicable.

\section{Competing interests}

The authors declare that they have no competing interests.

\footnotetext{
Author details

${ }^{1}$ School of Biomedical Engineering, University of British Columbia, Vancouver, BC, Canada. ${ }^{2}$ Center for Hip Health and Mobility, University of British Columbia, Vancouver, BC, Canada. ${ }^{3}$ Department of Medicine, University of British Columbia, Vancouver, BC, Canada. ${ }^{4}$ Arthritis Research Centre of Canada, Richmond, BC, Canada. ${ }^{5}$ Motion Analysis and Biofeedback Laboratory, University of British Columbia, Vancouver, BC, Canada. ${ }^{6}$ Department of Physical Therapy, University of British Columbia, Vancouver, BC, Canada. ${ }^{7}$ Department of Orthopaedics, University of British Columbia, 7/F, 2635 Laurel Street, Vancouver, BC V5Z1M9, Canada.
}

Received: 3 May 2021 Accepted: 26 October 2021

Published online: 23 November 2021

\section{References}

1. Ganz R, Parvizi J, Beck M, Leunig M, Nötzli H, Siebenrock KA. Femoroacetabular impingement: a cause for osteoarthritis of the hip. In: Clinical Orthopaedics and Related Research [Internet]. Lippincott Williams and Wilkins; 2003 [cited 2020 Oct 29]. p. 112-20. Available from: https://journ als.Iww.com/clinorthop/Fulltext/2003/12000/Femoroacetabular_Impin gement_A_Cause_for.13.aspx

2. Ganz R, Leunig M, Leunig-Ganz K, Harris WH. The etiology of osteoarthritis of the hip: an integrated mechanical concept. Clin Orthop Relat Res. 2008;466(2):264-72

3. Beck M, Kalhor M, Leunig M, Ganz R. Hip morphology influences the pattern of damage to the acetabular cartilage. Femoroacetabular impingement as a cause of early osteoarthritis of the hip. J Bone Jt Surg - Ser B [Internet]. 2005;87(7):1012-8. Available from: http://www.bjj.boneandjoi nt.org.uk/cgi/doi/10.1302/0301-620X.87B7.15203

4. Thomas GER, Palmer AJR, Batra RN, Kiran A, Hart D, Spector T, et al. Subclinical deformities of the hip are significant predictors of radiographic osteoarthritis and joint replacement in women. A 20 year longitudinal cohort study. Osteoarthr Cartil [Internet]. 2014;22(10):1504-10. Available from: https://doi.org/10.1016/j.joca.2014.06.038

5. Nicholls AS, Kiran A, Pollard TCB, Hart DJ, Arden CPA, Spector T, et al. The association between hip morphology parameters and nineteen-year risk of end-stage osteoarthritis of the hip: a nested case-control study. Arthritis Rheum. 2011;63(11):3392-400.

6. Agricola R, Heijboer MP, Bierma-Zeinstra SMA, Verhaar JAN, Weinans $\mathrm{H}$, Waarsing $\mathrm{JH}$. Cam impingement causes osteoarthritis of the hip: a nationwide prospective cohort study (CHECK). Ann Rheum Dis [Internet]. 2013;72(6):918-23 Available from: http://ard.bmj.com/lookup/doi/10. 1136/annrheumdis-2012-201643.

7. LaFrance R, Williams R, Madsen W, Maloney M, Drinkwater C, Giordano B. The prevalence of radiographic criteria of femoral acetabular impingement in patients undergoing hip arthroplasty surgery. Geriatr Orthop Surg Rehabil [Internet]. 2014;5(1):21-6 Available from: http://www. pubmedcentral.nih.gov/articlerender.fcgi?artid=3962054\&tool=pmcen trez\&rendertype=abstract.

8. Tanzer M, Noiseux N. Osseous abnormalities and early osteoarthritis: the role of hip impingement. In: Clinical Orthopaedics and Related Research [Internet]. Lippincott Williams and Wilkins; 2004 [cited 2020 Jun 17]. p. 170-7. Available from: http://journals.lww.com/00003086-20041 2000-00026

9. Enseki K, Harris-Hayes M, White DM, Cibulka MT, Woehrle J, Fagerson $\mathrm{TL}$, et al. Nonarthritic hip joint pain. J Orthop Sport Phys Ther [Internet]. 2014;44(6):A1-32 Available from: http://www.jospt.org/doi/10.2519/jospt. 2014.0302.

10. Hammoud S, Bedi A, Voos JE, Mauro CS, Kelly BT. The recognition and evaluation of patterns of compensatory injury in patients with mechanical hip pain. Sports Health [Internet]. 2014 Mar 11 [cited 2020 Jun 17];6(2):108-18. Available from: http://www.ncbi.nlm.nih.gov/pubmed/ 24587859.

11. Bedi A, Dolan M, Leunig M, Kelly BT. Static and dynamic mechanical causes of hip pain. Arthrosc J Arthrosc Relat Surg [Internet]. 2011;27(2):235-51 Available from: http://linkinghub.elsevier.com/retri eve/pii/S0749806310007267.

12. Kemp J, Grimaldi A, Heerey J, Jones D, Scholes M, Lawrenson P, et al. Current trends in sport and exercise hip conditions: intra-articular and extraarticular hip pain, with detailed focus on femoroacetabular impingement (FAl) syndrome, vol. 33. Best Practice and Research: Clinical Rheumatology. Bailliere Tindall Ltd; 2019. p. 66-87

13. Griffin DR, Dickenson EJ, O'Donnell J, Agricola R, Awan T, Beck M, et al. The Warwick agreement on femoroacetabular impingement syndrome (FAl syndrome): an international consensus statement. Br J Sports Med [Internet]. 2016;50(19):1169-76 Available from: http://bjsm.bmj.com/ lookup/doi/10.1136/bjsports-2016-096743.

14. Ackerman GP, McHugh MP, Bharam S. The impact of preoperative planning software on arthroscopic correction of femoroacetabular impingement. Orthop J Sport Med [Internet]. 2015;3(7_ suppl2):2325967115S0005. Available from: http://journals.sagepub.com/ doi/10.1177/2325967115S00056

15. Kennedy MJ, Lamontagne M, Beaulé PE. Femoroacetabular impingement alters hip and pelvic biomechanics during gait. Walking biomechanics of FAI. Gait Posture. 2009;30(1):41-4. 
16. Hunt MA, Gunether JR, Gilbart MK. Kinematic and kinetic differences during walking in patients with and without symptomatic femoroacetabular impingement. Clin Biomech [Internet]. 2013;28(5):519-23 Available from: https://doi.org/10.1016/j.clinbiomech.2013.05.002.

17. Brisson N, Lamontagne M, Kennedy MJ, Beaulé PE. The effects of cam femoroacetabular impingement corrective surgery on lower-extremity gait biomechanics. Gait Posture. 2013;37(2):258-63.

18. Rylander J, Shu B, Favre J, Safran M, Andriacchi T. Functional testing provides unique insights into the pathomechanics of femoroacetabular impingement and an objective basis for evaluating treatment outcome. J Orthop Res. 2013;31(9):1461-8.

19. Lamontagne M, Kennedy MJ, Beaulé PE. The effect of cam FAl on hip and pelvic motion during maximum squat. Clin Orthop Relat Res. 2009;467(3):645-50.

20. Bagwell J, Snibbe J, Gerhardt M, Powers CM. Hip kinematics and kinetics in persons with and without cam femoroacetabular impingement during a deep squat task. Clin Biomech [Internet]. 2016;31:87-92 Available from: https://doi.org/10.1016/j.clinbiomech.2015.09.016.

21. Ng KCG, Lamontagne M, Adamczyk AP, Rahkra KS, Beaulé PE. Patient-specific anatomical and functional parameters provide new insights into the pathomechanism of cam FAl. Clin Orthop Relat Res. 2015;473(4):1289-96.

22. King MG, Lawrenson PR, Semciw Al, Middleton KJ, Crossley KM. Lower limb biomechanics in femoroacetabular impingement syndrome: a systematic review and meta-analysis. Br J Sports Med. 2018;52(9):566-80.

23. Ng KCG, Rouhi G, Lamontagne M, Beaulé PE. Finite element analysis examining the effects of cam FAl on hip joint mechanical loading using subject-specific geometries during standing and maximum squat. HSS J. 2012;8(3):206-12.

24. Ng KCG, Lamontagne M, Labrosse MR, Beaulé PE. Hip joint stresses due to cam-type femoroacetabular impingement: a systematic review of finite element simulations. PLoS One 2016;11(1):1-19.

25. Audenaert EA, Peeters I, Vigneron L, Baelde N, Pattyn C. Hip morphological characteristics and range of internal rotation in femoroacetabular impingement. Am J Sports Med [Internet]. 2012;40(6):1329-36 Available from: http://journals.sagepub.com/doi/10.1177/0363546512441328.

26. Kubiak-Langer M, Tannast M, Murphy SB, Siebenrock KA, Langlotz F. Range of motion in anterior femoroacetabular impingement. Clin Orthop Relat Res [Internet]. 2007;PAP(458):117-24. Available from: http://content. wkhealth.com/linkback/openurl?sid=WKPTLP:landingpage\&an=00003 086-900000000-99029

27. Bedi A, Dolan M, Magennis E, Lipman J, Buly R, Kelly BT. Computerassisted modeling of osseous impingement and resection in femoroacetabular impingement. Arthrosc - J Arthrosc Relat Surg. 2012;28(2):204-10.

28. Audenaert EA, Mahieu P, Pattyn C. Three-dimensional assessment of cam engagement in femoroacetabular impingement. Arthrosc - J Arthrosc Relat Surg [Internet]. 2011;27(2):167-71 Available from: https://doi.org/10. 1016/j.arthro.2010.06.031.

29. Kopec JA, Cibere J, Li LC, Zhang C, Barber M, Qian H, et al. Relationship between physical activity and hip pain in persons with and without cam or pincer morphology: a population-based case-control study. Osteoarthr Cartil. 2017 Jul 1;25(7):1055-61.

30. Guo Y, Zhang H, Qian H, Wilson DR, Wong H, Barber M, et al. Association of femoroacetabular impingement and delayed gadolinium-enhanced magnetic resonance imaging of cartilage: a population-based study. Arthritis Care Res [Internet]. 2018 Aug 1 [cited 2020 Jun 17];70(8):1 160-8. Available from: http://doi.wiley.com/10.1002/acr.23463

31. Ratzlaff C, Zhang C, Korzan J, Josey L, Wong H, Cibere J, et al. The validity of a non-radiologist reader in identifying cam and pincer femoroacetabular impingement (FAl) using plain radiography. Rheumatol Int [Internet]. 2016 Mar 1 [cited 2020 Jun 17];36(3):371-6. Available from: https://link. springer.com/article/10.1007/s00296-015-3361-7

32. Nötzli HP, Wyss TF, Stoecklin CH, Schmid MR, Treiber K, Hodler J. The contour of the femoral head-neck junction as a predictor for the risk of anterior impingement. J Bone Joint Surg Br [Internet]. 2002 May 1 [cited 2020 Jun 17];84-B(4):556-60. Available from: https://online.boneandjoint. org.uk/doi/abs/10.1302/0301-620X.84B4.0840556

33. Tannast M, Siebenrock KA, Anderson SE. Femoroacetabular impingement: radiographic diagnosis-what the radiologist should know. Am J Roentgenol. 2007;188(6):1540-52.
34. Wiberg G. Studies on dysplastic acetabula and congenital subluxation of the hip joint with special reference to the complication of osteo-arthritis. J Am Med Assoc [Internet]. 1940 Jul 6 [cited 2020 Jun 17];115(1):81. Available from: https://www.worldcat.org/title/studies-on-dysplastic-aceta bula-and-congenital-subluxation-of-the-hip-joint-with-special-referenceto-the-complication-of-osteoarthritis/oclc/186329387

35. Monazzam S, Bomar JD, Cidambi K, Kruk P, Hosalkar H. Lateral centeredge angle on conventional radiography and computed tomography hip. Clin Orthop Relat Res. 2013;471(7):2233-7.

36. Wu G, Siegler S, Allard P, Kirtley C, Leardini A, Rosenbaum D, et al. ISB recommendation on definitions of joint coordinate system of various joints for the reporting of human joint motion - Part I: ankle, hip, and spine. Vol. 35, Journal of Biomechanics. Elsevier Ltd; 2002. p. 543-548.

37. Kroon DJ. Segmentation of the mandibular canal in cone-beam CT data [Internet]. 2011 [cited 2020 Jun 17]. 154 p. Available from: https:// research.utwente.nl/en/publications/segmentation-of-the-mandi bular-canal-in-cone-beam-ct-data

38. Grood ES, Suntay WJ. A joint coordinate system for the clinical description of three-dimensional motions: application to the knee. J Biomech Eng [Internet]. 1983 [cited 2020 17];105(2):136-44. Available from: https:// pubmed.ncbi.nlm.nih.gov/6865355/

39. Wyss TF, Clark JM, Weishaupt D, Nötzli HP. Correlation between internal rotation and bony anatomy in the hip. Clin Orthop Relat res [internet]. 2007:460(460):152-158. Available from: http://www.ncbi.nlm.nih.gov/ pubmed/17290151.

40. Buchan LL, Zhang H, Konan S, Heaslip I, Ratzlaff CR, Wilson DR. Open-MRI measures of cam intrusion for hips in an anterior impingement position relate to acetabular contact force. J Orthop Res [Internet]. 2016 Feb 1 [cited 2020 Jun 17];34(2):205-16. Available from: https://pubmed.ncbi. nlm.nih.gov/26241132/

41. Kapron AL, Aoki SK, Peters CL, Anderson AE. Subject-specific patterns of femur-labrum contact are complex and vary in asymptomatic hips and hips with femoroacetabular impingement. Clin Orthop Relat Res [Internet]. 2014 Dec 1 [cited 2021 Jan 14];472(12):3912-22. Available from: https://link.springer.com/article/10.1007/s11999-014-3919-9

42. Kapron AL, Aoki SK, Peters CL, Maas SA, Bey MJ, Zauel R, et al. Accuracy and feasibility of dual fluoroscopy and model-based tracking to quantify in vivo hip kinematics during clinical exams. J Appl Biomech. 2014;30(3):461-70.

43. Chegini S, Beck M, Ferguson SJ. The effects of impingement and dysplasia on stress distributions in the hip joint during sitting and walking: a finite element analysis. J Orthop Res. 2009;27(2):195-201.

44. Jorge JP, Simões FMF, Pires EB, Rego PA, Tavares DG, Lopes DS, et al. Finite element simulations of a hip joint with femoroacetabular impingement. Comput Methods Biomech Biomed Engin [Internet]. 2014;17(11):1275-84 Available from: http://www.tandfonline.com/doi/abs/10.1080/10255842. 2012.744398.

45. Hellwig FL, Tong J, Hussell JG. Hip joint degeneration due to cam impingement: a finite element analysis. Comput Methods Biomech Biomed Engin [Internet]. 2016;19(1):41-8 Available from: https://doi.org/ 10.1080/10255842.2014.983490.

46. Gala L, Khanna V, Rakhra KS, Beaulé PE. Does the femoral head/neck contour in the skeletally mature change over time? J Hip Preserv Surg [Internet]. 2016 Oct 1 [cited 2020 Jun 17];3(4):333-7. Available from: https://academic.oup.com/jhps/article/3/4/333/2525439

47. Steppacher SD, Tannast M, Ganz R, Siebenrock KA. Mean 20-year followup of bernese periacetabular osteotomy. Clin Orthop Relat Res [Internet]. 2008 [cited 2020 Jun 17];466(7):1633-44. Available from: https://pubmed. ncbi.nlm.nih.gov/18449617/

\section{Publisher's Note}

Springer Nature remains neutral with regard to jurisdictional claims in published maps and institutional affiliations. 